21st Particles and Nuclei International Conference (PANIC 2017)

International Journal of Modern Physics: Conference Series

Vol. 46 (2018) 1860047 (5 pages)

(C) The Author(s)

DOI: $10.1142 / \mathrm{S} 2010194518600479$

\title{
Dark Photon Search with PADME at LNF
}

\author{
Gabriele Piperno (for the PADME collaboration*) \\ Research Division, INFN Laboratori Nazionali di Frascati, \\ Via E. Fermi, 40 Frascati (Rome), 00044, Italy \\ gabriele.piperno@lnf.infn.it
}

Published 3 May 2018

\begin{abstract}
Dark Matter elusiveness could be explained by speculating that it lives in a separate sector with respect to the Standard Model and that interacts with it only by means of messengers. The simplest model foresees just one messenger: a, possibly massive, vector boson given by a new U(1) symmetry. This mediator can faintly mix with the photon and, hence, interact with SM charged particles, seeing an effective charge equal to $\varepsilon e$, with e SM charge. The PADME experiment, hosted at Laboratori Nazionali di Frascati, is designed to search for such kind of particle, looking for its production in $e^{+} e^{-}$annihilations. Exploiting the DA $\Phi$ NE linac, the collaboration aims to collect $10^{13}$ positrons on target by the end of 2018, reaching a sensitivity of $\sim 10^{-3}$ for masses up to $23.7 \mathrm{MeV}$.
\end{abstract}

Keywords: Dark Photon; Dark Matter; Missing mass search.

\section{Introduction}

Since many decades there is a problem in particle physics that is still waiting for a solution: the Dark Matter (DM) identification. By many indications we know that it must exist, but there are no clear and incontrovertible evidences of it. A possible explanation is that DM "lives" in a Dark Sector (DS) and interacts with the Standard Model (SM) particles only by means of mediators. The simplest model

* G. Chiodini, P. Creti, F. Oliva, V. Scherini (INFN Lecce); A.P. Caricato, M. Martino, G. Maruccio, A. Monteduro, S. Spagnolo (INFN Lecce e Dip. di Matematica e Fisica, Università del Salento); P. Albicocco, R. Bedogni, F. Bossi, B. Buonomo, R. de Sangro, G. Finocchiaro, L.G. Foggetta, A. Ghigo, P. Gianotti, M. Palutan, G. Piperno, I. Sarra, B. Sciascia, T. Spadaro, E. Spiriti, C. Taruggi, E. Vilucchi (INFN Laboratori Nazionali di Frascati); F. Ameli, F. Ferrarotto, E. Leonardi, F. Safai Tehrani, P. Valente (INFN Roma1); S. Fiore (INFN Roma1 e ENEA); G.C. Organtini, M. Raggi (INFN Roma1 e Dip. di Fisica, "Sapienza" Università di Roma); L. Tsankov (University of Sofia "St. Kl. Ohridski"); G. Georgiev, V. Kozhuharov (University of Sofia "St. Kl. Ohridski" and INFN Laboratori Nazionali di Frascati).

This is an Open Access article published by World Scientific Publishing Company. It is distributed under the terms of the Creative Commons Attribution 4.0 (CC-BY) License. Further distribution of this work is permitted, provided the original work is properly cited. 
introduces a new U(1) symmetry with its, possibly massive, vector boson, indicated with the symbol $A^{\prime 1,2}$. The SM particles are neutral under this symmetry, while the mediator can faintly mix with the standard photon and couple to SM particles with an effective charge $\varepsilon e$, where $\varepsilon$ is the mixing constant and $e$ is the electromagnetic charge of the SM particle. For this reason $A^{\prime}$ is generally called Dark Photon (DP).

This approach results to be extremely predictive and simple since it requires to introduce only two new parameters to describe the DP: its mass $m_{A^{\prime}}$ and the constant $\varepsilon$.

In addition, depending on the selected model, such a particle could justify, totally or partially, the observed difference ${ }^{3}$ between the measured and the expected value of the muon anomalous magnetic moment $(g-2)_{\mu} \cdot{ }^{4}$

If there are no DM particles with mass $m_{D M}$ such that $m_{D M} \leq \frac{1}{2} m_{A^{\prime}}$, the $A^{\prime}$ will decay only into SM particles (visible decays). In the other case, the $A^{\prime}$ will decay predominantly into DS particles (invisible decays), suppressing by a factor $\varepsilon^{2}$ the SM decays. In both cases, the DP coming from this simple model has been discarded as the only responsible of the $(g-2)_{\mu}$ discrepancy. ${ }^{5,6}$

To have a complete scenario of the research status and of the experimental techniques presently used see Refs. 7 and 8 and references therein.

\section{The experimental technique}

Hosted at the Laboratori Nazionali di Frascati and placed in the newly redesigned Beam Test Facility ${ }^{9}$, the PADME (Positron Annihilation into Dark Matter Experiment) 10,11 experiment has as main goal the search for the DP, looking for the reaction:

$$
e^{+} e^{-} \rightarrow A^{\prime} \gamma
$$

The needed positrons are produced and accelerated up to $550 \mathrm{MeV}$ by the Frascati linear accelerator, while the electrons come from a diamond active target. If an $A^{\prime}$ is produced, the event would appear as a single photon with missing energy in the detector due to the DP, that leaves the experiment undetected. Hence, knowing the initial positron and electron quadri-momentum $\left(\vec{P}_{e^{+}}, 550 \mathrm{MeV}\right.$ in the beam direction and $\vec{P}_{e^{-}}=\overrightarrow{0}$, respectively) and measuring the recoil photon in the final state $\left(\vec{P}_{\gamma}\right)$, it is possible to evaluate the squared missing mass as:

$$
M_{m i s s}^{2}=\left(\vec{P}_{e^{-}}+\vec{P}_{b e a m}-\vec{P}_{\gamma}\right)^{2} .
$$

Taking as reference Fig. 1, from right to left, the detector components are:

- Diamond active target $\left(2 \times 2 \mathrm{~cm}^{2}\right.$ area for $100 \mu \mathrm{m}$ thickness $)$. It allows to measure the beam intensity and position $(\approx 5 \mathrm{~mm}$ precision $)$, thanks to vertical and horizontal graphitic strips. The target low $\mathrm{Z}$ is to reduce the bremsstrahlung background, while the small thickness is to lower the probability of positron multiple interactions. 


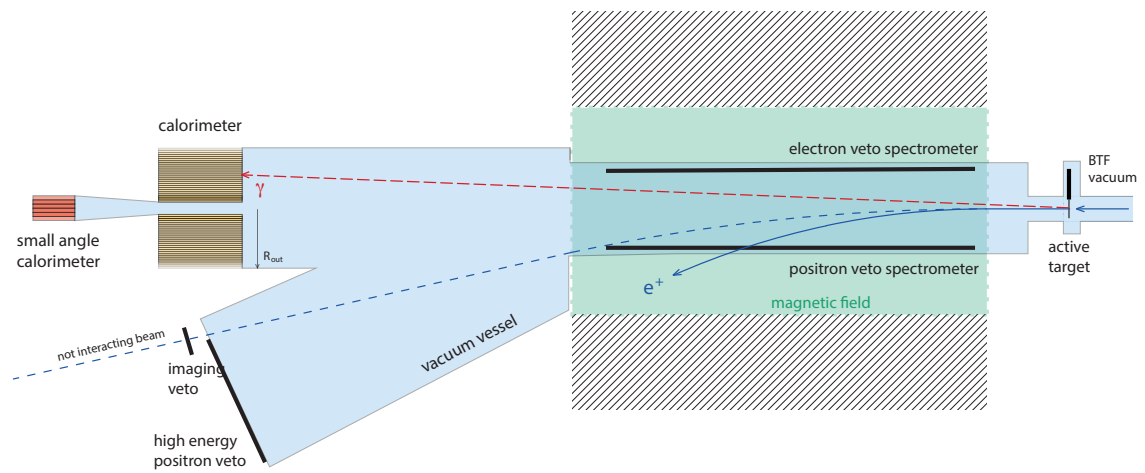

Fig. 1. Schematic representation of the PADME detector, with the $e^{+}$beam coming from the right, incident on the active target, passing through the magnetic dipole (inside which there are the $e^{+} / e^{-}$vetoes), and exiting near the high-energy positron veto. The ECal and the SAC are placed $3 \mathrm{~m}$ downstream of the target.

- Magnetic dipole (1 m length for $23 \mathrm{~cm}$ gap). It is placed $20 \mathrm{~cm}$ after the target and provides $\mathrm{a} \approx 0.5 \mathrm{~T}$ field. It is needed to bend the beam towards the exit and to send particles that loose energy, typically for bremsstrahlung, to the veto's detectors.

- Positrons/electrons (high-energy) veto. This component of the detector is subdivided into two parts: one inside the magnetic dipole for positrons and electrons (produced in the beam/target interaction) and one close to the beam exit, for positrons that loose only a small amount of energy. It is composed of plastic scintillator bars with dimensions $1 \times 1 \times 16 \mathrm{~cm}^{3}$. The arrays inside the dipole are $1 \mathrm{~m}$ long, while the high-energy positron veto is $0.5 \mathrm{~m}$ long.

- Electromagnetic calorimeter (ECal). It consists of 616 bismuth germanate (BGO) crystals of dimensions $2.1 \times 2.1 \times 23.0 \mathrm{~cm}^{3}$ arranged in a cylindrical shape of $\approx 60 \mathrm{~cm}$ diameter. It also presents a central squared hole five crystals wide to let the bremsstrahlung radiation to pass and to be detected by a faster small angle calorimeter placed behind it; this is necessary because the BGO has a long scintillation light decay time of $300 \mathrm{~ns}$ and would be continuously "blinded" by the event rate. The expected energy resolution is $\approx \frac{2 \%}{\sqrt{E}} 12$ and the angular coverage is $(20,95)$ mrad.

- Small Angle Calorimeter (SAC). Made of 25 lead difluoride $\left(\mathrm{PbF}_{2}\right)$ elements of $3 \times 3 \times 14 \mathrm{~cm}^{3}$, its purpose is to veto events with a bremsstrahlung photon. Being based on Cherenkov detectors, it has a dead time of about $3 \mathrm{~ns}$ and is able to stand event rates $\sim 10$ times higher than the BGO. The given angular coverage is $(0,20) \mathrm{mrad}$.

The expected signature of signal events consists of a single $\gamma$ in the ECal and no other hits in the detector (veto and SAC). With a beam energy of $550 \mathrm{MeV}$, the maximum DP mass that can be explored is $23.7 \mathrm{MeV}$. 


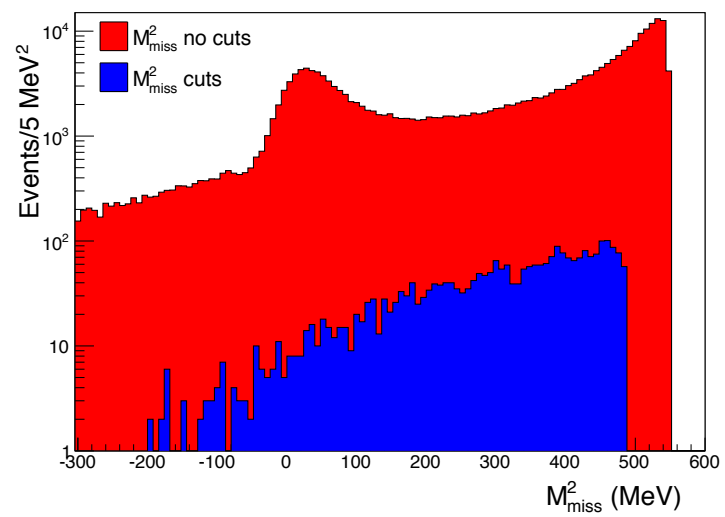

Fig. 2. Background comparison before (red) and after (blue) event selection.

\section{Backgrounds and sensitivity}

There are two main SM sources of background for the DP search: the bremsstrahlung $\left(e^{+} N \rightarrow e^{+} N \gamma\right)$ and the annihilation into two $\gamma \mathrm{s}$, possibly with the emission of a initial state radiation $\left(e^{+} e^{-} \rightarrow \gamma \gamma(\gamma)\right)$. The former is important for higher values of $M_{m i s s}^{2}$ and is reduced using the SAC. The latter, instead, is important for medium and small $M_{m i s s}^{2}$ and is cut down by the calorimeter granularity and geometry. In addition, if the clusters in the ECal are temporally too close each other, also the pile-up could represent an important source of background, but this can be kept under control maintaining the beam intensity around 5000 positrons per bunch. In Fig. 2 it is presented the comparison between the background without any applied selection (in red) and the one obtained requiring only one cluster in the ECal (with

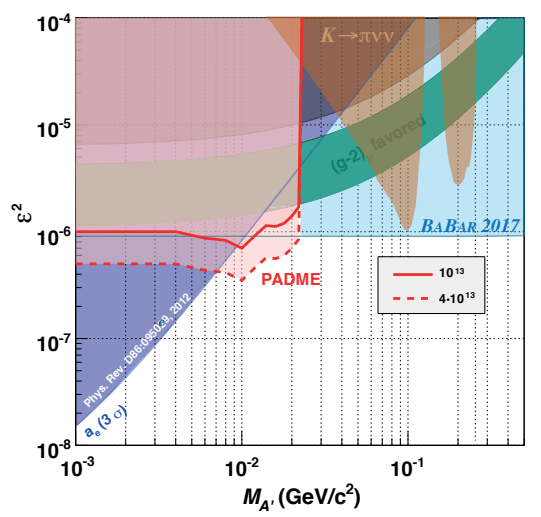

Fig. 3. PADME sensitivity for an invisibly decaying $A^{\prime}$ for different collected statistics with beam energy of $550 \mathrm{MeV}$. 
an energy that depends on $m_{A^{\prime}}$ ), no hits in the veto and no photons with energy $>50 \mathrm{MeV}$ in the $\mathrm{SAC}$ (in blue).

The sensitivity of the experiment to the DP has been evaluated simulating 2.5. $10^{10} 550 \mathrm{MeV}$ Positrons On Target (POT) and, then, extrapolating the result to the desired number of events. In Fig. 3 are shown the expected sensitivities for $10^{13}$ and $4 \cdot 10^{13}$ POT in the squared coupling constant versus mass plane. With these numbers of events, it is possible to scan, in a model independent way, since no assumptions are made on the $A^{\prime}$ decay chain, $\varepsilon^{2}$ of the order of $10^{-6}$ for a DP that decays in invisible.

\section{Conclusions}

In the last years the search for DP signals is growing in importance as a possible solution to the DM detection problem. It can also explain, partially or entirely depending on the selected model, the muon anomalous magnetic moment discrepancy between the theoretical expectations and the experimental results.

In this context, the PADME collaboration aims to collect $10^{13}$ POT by the end of 2018, probing, in a model independent way, the existence of a DP with masses up to $23.7 \mathrm{MeV}$ and coupling constants $\varepsilon$ of $10^{-3}$ or lower, that decays into DS particles.

\section{References}

1. B. Holdom., Phys. Lett. B 166 (1986) 196.

2. P. Galison e A. Manohar, Phys. Lett. B 136 (1984) 279.

3. G. W. Bennett et al., Phys. Rev. D 73 (2006) 072003.

4. M. Pospelov, Phys. Rev. D 80 (2009) 095002.

5. M. Ablikim et al., Phys. Lett. B 774 (2017) 252.

6. J. P. Lees et al., Phys. Rev. Lett. 119 (2017) 131804.

7. M. Raggi e V. Kozhuharov, Riv. Nuovo Cim. 38 (2015) 449.

8. M. Battaglieri et al., arXiv:1707.04591

9. G. Mazzitelli et al., Nucl. Instrum. Meth. A 515, 524 (2003).

10. M. Raggi e V. Kozhuharov, Adv. High Energy Phys. 2014 (2014) 959802.

11. M. Raggi, V. Kozhuharov e P. Valente, EPJ Web Conf. 96 (2015) 01025.

12. M. Raggi et al., Nucl. Instrum. Meth. A 862 (2017) 31. 